\title{
RADIOGRAPHIC MEASUREMENTS OF METACARPOPHALANGEAL LENGTHS IN JAPANESE CHILDREN
}

\author{
Shinya MatsuUra and Tadashi KaJII \\ Department of Pediatrics, Yamaguchi University School of Medicine, \\ Ube 755, Japan
}

\begin{abstract}
Summary The lengths of 19 tubular bones of the hand were measured on radiographs of 1,585 Japanese children aged 0 to 17 years. The means and standard deviations related to age and sex were calculated and presented in tables. Using the standards, examples of the metacarpophalangeal pattern profile analysis are presented.
\end{abstract}

Key Words metacarpals, phalanges, hand, Japanese children

\section{INTRODUCTION}

The lengths of metacarpals and phalanges are altered in a wide variety of multiple malformation syndromes. Thus, metacarpophalangeal pattern profile (MCPP) analysis, in which the plus or minus deviations of the bone length from the age- and sex-related mean values are displayed on a graph, has been increasingly used as a means of defining hand malformations in known syndromes (Al-Awadi et al., 1988; Butler et al., 1985a, 1985b, 1986, 1987a, 1987b, 1988), or as a tool in the diagnosis of patients with hand malformations (Tonoki et al., 1988). It is also a powerful tool in the identification of affected individuals in pedigree studies of malformation syndromes (Poznanski et al., 1972, 1983).

There is a dearth of data for the lengths of hand tubular bones in Japanese children (Hasue et al., 1955; Kato et al., 1955; Kunishima, 1965). In view of this, we carried out such measurements in 1,585 normal Japanese children of the ages 0 to 17 years.

\section{MATERIALS AND METHODS}

Hand radiographs of 1,585 normal infants and children of both sexes, aged 0 to 17 years, were studied (Table 1). They included 1) 481 infants and children of up to 15 years of age, who visited the Department of Orthopedics, Nagoya University, and other orthopedic clinics in the Tokai area, for accidental injuries during

Received April 17, 1989; revised version received April 24, 1989; Accepted April 27, 1989 
Table 1. Distribution of the materials by age.

\begin{tabular}{rcccccccc}
\hline \multirow{2}{*}{$\begin{array}{c}\text { Age } \\
\text { yr) }\end{array}$} & \multicolumn{3}{c}{ Boys } & & \multicolumn{3}{c}{ Girls } & Total \\
\cline { 2 - 3 } \cline { 5 - 6 } & Nagoya & Saga & Nagasaki & Total & Nagoya & Saga & Nagasaki & \\
\hline 0 & 15 & & & 15 & 15 & & & 15 \\
1 & 12 & & & 12 & 13 & & & 13 \\
2 & 15 & & & 15 & 12 & & & 12 \\
3 & 21 & & & 21 & 9 & & & 9 \\
4 & 27 & & 24 & 51 & 21 & & 27 & 48 \\
5 & 12 & & 38 & 50 & 13 & & 36 & 49 \\
6 & 14 & 36 & & 50 & 11 & 39 & & 50 \\
7 & 19 & 31 & & 50 & 15 & 39 & & 54 \\
8 & 13 & 37 & & 50 & 11 & 39 & & 50 \\
9 & 20 & 45 & & 65 & 14 & 52 & & 66 \\
10 & 13 & 32 & 4 & 51 & 11 & 51 & & 62 \\
11 & 17 & 45 & & 62 & 14 & 30 & 6 & 50 \\
12 & 16 & 34 & & 50 & 11 & 49 & & 60 \\
13 & 20 & 29 & & 49 & 27 & 25 & & 52 \\
14 & 10 & 41 & & 51 & 15 & 37 & & 52 \\
15 & 9 & 39 & 3 & 51 & 14 & 36 & & 50 \\
16 & & 50 & & 50 & & 47 & & 47 \\
17 & & 50 & & 50 & & 53 & & 53 \\
\hline Total & 255 & 469 & 69 & 793 & 226 & 497 & 69 & 792 \\
\hline
\end{tabular}

the period from 1976 to 1983, and were judged otherwise normal (Sugiura, 1985); 2) 966 children, 6 to 17 years of age, drawn from a primary school, a junior high school and a senior high school in Ogi, Saga Prefecture, collected between 1982 and 1985 (part of Ogi Longitudinal Growth Study projected by T. Akiyoshi); and 3) 138 children mainly consisting of those who attended two kindergartens in Nomozaki, Nagasaki Prefecture, in 1978 and 1979 (Takai and Akiyoshi, 1983).

The infants and children were grouped according to sex and yearly age intervals. Those at the 0 to 3 year age range had 9 to 21 infants and children in each sex and age group, while those at the 4 to 17 year range consisted of 49 to 66 children in each group (Table 1).

All hand radiographs were taken in the postero-anterior position with uniform focal-tube distance of $100 \mathrm{~cm}$. Of the 1,585 infants and children radiographs of the right hand were used for study in 1,146 subjects, while those of the left hand were studied in the remaining 439 subjects. No appreciable difference between the right and left hands was noted in 42 children whose radiographs of both hands were available. Figure 1 shows measurements of bone lengths. Measure- 

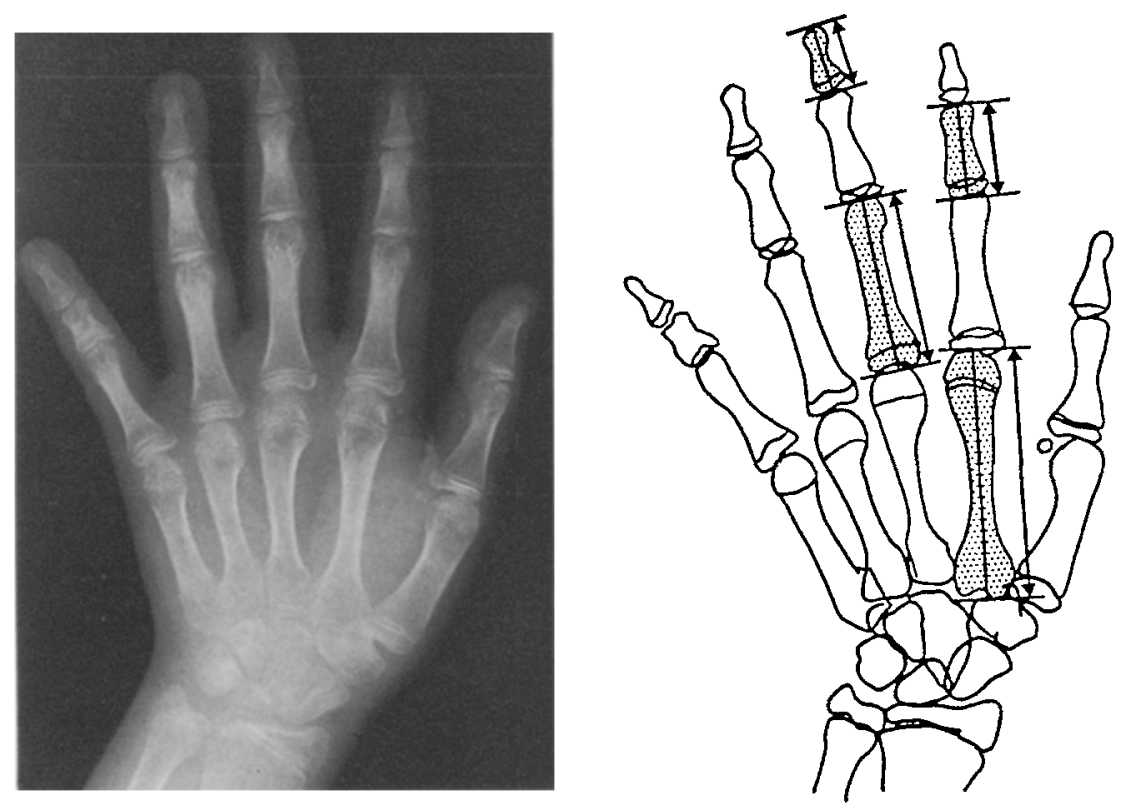

Fig. 1. Hand radiograph of a 16-year-old patient with the Turner syndrome with a 45,X karyotype. Length measurements of the metacarpals, the proximal, middle and distal phalanges made on the proximal-to-distal axis including the epiphysis when separate.

ments were made to nearest $0.1 \mathrm{~mm}$ with a Digimatic Caliper CD-15A (Mitutoyo) on the proximal-to-distal axial lengths of the 19 tubular bones of the hand. The tubular bones studied included the metacarpals and the proximal, middle and distal phalanges. All measurements were carried out by one of the authors (S.M.) and recorded by an assistant. For details of measurement, the reader is referred to Garn et al. (1972).

The data thus obtained were analyzed essentially according to the method as described previously (Igarashi and Kajii, 1988). Briefly, all computations were performed by an Epson PC-286 personal computer using programs written in Lotus 1-2-3 and Audemain Kagaku Keisan Forms. The raw data for each sex and age group were used to calculate the means, standard deviations, and minimal and maximal values. Exceptional, outlying values were rejected referring to the expected extreme values for a normal distribution of the estimated mean and standard deviation. The expected extreme values were calculated at the $1 \%$ level referring to Smirnoff's constant. Thus, of a total of 30,115 measurements, $107(0.4 \%)$ derived from 63 children were disregarded.

\section{RESULTS}

Table 2 shows the means and standard deviations for the lengths of 19 tubular 
Table 2A. Means and standard deviations (S.D.) for the lengths of the 19 tubu-

\begin{tabular}{|c|c|c|c|c|c|c|c|c|c|}
\hline \multirow{2}{*}{\multicolumn{2}{|c|}{ Bones }} & \multicolumn{2}{|c|}{0} & \multicolumn{2}{|c|}{1} & \multicolumn{2}{|c|}{2} & \multicolumn{2}{|c|}{3} \\
\hline & & Mean & S.D. & Mean & S.D. & Mean & S.D. & Mean & S.D. \\
\hline \multirow[t]{5}{*}{ Distal } & 5 & 5.2 & 0.6 & 5.9 & 0.6 & 7.2 & 0.8 & 8.6 & 1.4 \\
\hline & 4 & 6.5 & 0.6 & 7.3 & 0.8 & 9.1 & 1.0 & 10.4 & 1.2 \\
\hline & 3 & 6.3 & 0.5 & 7.1 & 0.6 & 9.0 & 1.0 & 10.1 & 1.2 \\
\hline & 2 & 5.6 & 0.5 & 5.8 & 0.7 & 7.4 & 0.8 & 9.1 & 1.1 \\
\hline & 1 & 7.5 & 0.6 & 9.3 & 1. 1 & 11.3 & 0.9 & 12.6 & 1.1 \\
\hline \multirow[t]{4}{*}{ Middle } & 5 & 6.0 & 0.9 & 7.4 & 1.0 & 8.4 & 1.0 & 9.5 & 1.4 \\
\hline & 4 & 9.6 & 1.0 & 11.6 & 1.1 & 13.5 & 1.1 & 14.8 & 1.5 \\
\hline & 3 & 10.1 & 0.9 & 11.6 & 0.9 & 13.8 & 1.2 & 15.5 & 1.6 \\
\hline & 2 & 8.2 & 0.8 & 9.5 & 0.6 & 11.0 & 0.8 & 12.9 & 1.2 \\
\hline \multirow[t]{5}{*}{ Proximal } & 5 & 11.5 & 1.5 & 14.2 & 1.3 & 16.8 & 1.3 & 18.7 & 1.7 \\
\hline & 4 & 14.9 & 1.9 & 18.7 & 1.8 & 22.0 & 1.7 & 24.2 & 2. 0 \\
\hline & 3 & 15.8 & 1.8 & 20.1 & 1.8 & 23.1 & 2.0 & 25.7 & 2.0 \\
\hline & 2 & 13.8 & 2.3 & 17.8 & 1.6 & 20.8 & 1.8 & 23.1 & 1.8 \\
\hline & 1 & 10.2 & 1.2 & 12.6 & 1.2 & 14.4 & 1.5 & 16.6 & 1.7 \\
\hline \multirow[t]{5}{*}{ Metacarpal } & 5 & 15.3 & 1.7 & 18.8 & 1.8 & 23.1 & 2.3 & 26.7 & 2.1 \\
\hline & 4 & 16.7 & 2.0 & 21.3 & 2.1 & 25.9 & 2.7 & 30.1 & 2.9 \\
\hline & 3 & 18.9 & 2.1 & 23.9 & 2.4 & 29.4 & 2.8 & 33.3 & 2.4 \\
\hline & 2 & 20.2 & 2.4 & 25.1 & 3.3 & 31.7 & 2.8 & 35.4 & 2.3 \\
\hline & 1 & 12.8 & 1.5 & 15.7 & 1.6 & 19.6 & 2.0 & 23.1 & 1.6 \\
\hline \multirow[t]{5}{*}{ Distal } & 5 & 5.5 & 1.5 & 6.3 & 0.9 & 8.3 & 0.4 & 8.7 & 1.0 \\
\hline & 4 & 6.2 & 0.5 & 8.0 & 1.1 & 9.7 & 0.9 & 10.4 & 1.0 \\
\hline & 3 & 6.2 & 0.5 & 7.9 & 0.9 & 9.4 & 0.8 & 10.2 & 0.9 \\
\hline & 2 & 5.3 & 0.6 & 6.7 & 0.8 & 8.5 & 0.5 & 9.3 & 1.1 \\
\hline & 1 & 7.3 & 1.2 & 10.1 & 0.8 & 11.6 & 1.0 & 12.1 & 1.3 \\
\hline \multirow[t]{4}{*}{ Middle } & 5 & 6.1 & 0.5 & 7.4 & 1.0 & 9.0 & 1.2 & 9.5 & 1.5 \\
\hline & 4 & 9.4 & 1.4 & 11.9 & 1.2 & 13.9 & 1.2 & 14.5 & 1.5 \\
\hline & 3 & 9.8 & 1.2 & 12.5 & 1.2 & 14.4 & 1.3 & 15.3 & 1.5 \\
\hline & 2 & 8.3 & 0.8 & 10.1 & 0.8 & 11.8 & 1.2 & 12.9 & 1.4 \\
\hline \multirow[t]{5}{*}{ Proximal } & 5 & 11.1 & 1.6 & 15.1 & 1.3 & 17.0 & 1.2 & 18.1 & 1.6 \\
\hline & 4 & 14.6 & 2.4 & 19.6 & 1.2 & 22.1 & 1.3 & 23.4 & 2. 1 \\
\hline & 3 & 15.5 & 2.5 & 20.8 & 1.2 & 23.6 & 1.6 & 25.0 & 2.3 \\
\hline & 2 & 13.9 & 2.2 & 18.7 & 1.1 & 21.0 & 1.4 & 22.6 & 1.9 \\
\hline & 1 & 9.7 & 1.4 & 12.7 & 1.1 & 15.5 & 1.4 & 16.4 & 1.6 \\
\hline \multirow[t]{5}{*}{ Metacarpal } & 5 & 14.5 & 1.8 & 20.3 & 2.5 & 23.3 & 2.1 & 26.5 & 2.7 \\
\hline & 4 & 16.3 & 2.3 & 22.7 & 3.1 & 26.2 & 1.8 & 29.9 & 3.0 \\
\hline & 3 & 18.5 & 2.5 & 25.3 & 3.0 & 29.4 & 2.2 & 33.5 & 3.4 \\
\hline & 2 & 19.3 & 2.8 & 27.3 & 3.3 & 31.5 & 2.2 & 35.4 & 3.3 \\
\hline & 1 & 12.6 & 1.9 & 17.4 & 2.0 & 20.5 & 2.1 & 23.0 & 2.9 \\
\hline
\end{tabular}


lar bones related to age (0-8 years) and sex (upper half, boys; lower half, girls).

\begin{tabular}{|c|c|c|c|c|c|c|c|c|c|}
\hline \multicolumn{2}{|c|}{4} & \multicolumn{2}{|c|}{5} & \multicolumn{2}{|c|}{6} & \multicolumn{2}{|c|}{7} & \multicolumn{2}{|c|}{8} \\
\hline Mean & S.D. & Mean & S.D. & Mean & S.D. & Mean & S.D. & Mean & S.D. \\
\hline \multicolumn{10}{|l|}{ Boys } \\
\hline 9.4 & 0.8 & 10.3 & 0.7 & 10.8 & 0.6 & 11.5 & 0.9 & 12.3 & 1.0 \\
\hline 10.9 & 0.8 & 11.9 & 0.8 & 12.3 & 0.8 & 13.1 & 0.9 & 14.2 & 1.1 \\
\hline 10.6 & 0.8 & 11.6 & 0.8 & 12.0 & 0.7 & 12.8 & 0.8 & 13.8 & 1.1 \\
\hline 9.7 & 0.9 & 10.7 & 0.8 & 11.2 & 0.7 & 11.7 & 0.7 & 12.8 & 1.1 \\
\hline 13.1 & 0.9 & 14.0 & 1.0 & 14.8 & 1.0 & 15.6 & 1.2 & 17.0 & 1.3 \\
\hline 10.5 & 1.0 & 11.1 & 1.3 & 11.5 & 1.3 & 12.1 & 1.4 & 13.5 & 1.3 \\
\hline 15.6 & 1.0 & 16.6 & 1.2 & 17.2 & 1.2 & 17.9 & 1.3 & 19.5 & 1.3 \\
\hline 16.4 & 1.0 & 17.4 & 1.2 & 18.1 & 1.2 & 18.9 & 1.3 & 20.5 & 1.4 \\
\hline 13.6 & 0.9 & 14.6 & 1.1 & 15.2 & 1.1 & 15.9 & 1.2 & 17.2 & 1.3 \\
\hline 19.3 & 1.1 & 20.6 & 1.3 & 21.6 & 1.3 & 22.4 & 1.6 & 24.2 & 1.5 \\
\hline 25.0 & 1.3 & 26.6 & 1.5 & 28.1 & 1.6 & 29.0 & 2.0 & 31.1 & 1.5 \\
\hline 26.9 & 1.5 & 28.3 & 2.1 & 30.2 & 1.9 & 31.1 & 2.1 & 33.2 & 1.7 \\
\hline 23.9 & 1.4 & 25.4 & 1.5 & 27.0 & 1.7 & 27.7 & 1.9 & 30.1 & 1.9 \\
\hline 17.5 & 1.1 & 18.7 & 1.4 & 19.7 & 1.6 & 20.5 & 1.7 & 22.5 & 1.7 \\
\hline 28.7 & 2.2 & 31.3 & 2.2 & 33.4 & 2.5 & 35.0 & 2. 3 & 38.5 & 2. 3 \\
\hline 31.7 & 2.4 & 34.5 & 2.2 & 36.5 & 2.7 & 38.4 & 2.5 & 41.9 & 2.4 \\
\hline 35.5 & 2.7 & 38.4 & 2.5 & 41.0 & 3.0 & 42.8 & 2.9 & 46.7 & 2.5 \\
\hline 38.0 & 2.6 & 40.7 & 2.3 & 43.5 & 3.0 & 45.3 & 3.1 & 49.2 & 2.7 \\
\hline 24.7 & 1.8 & 26.6 & 2.0 & 28.6 & 2.0 & 29.9 & 2.3 & 33.1 & 2.2 \\
\hline \multicolumn{10}{|l|}{ Girls } \\
\hline 9.8 & 0.7 & 10.4 & 0.8 & 11.1 & 1.0 & 11.8 & 1.0 & 12.2 & 1.0 \\
\hline 11.0 & 0.8 & 11.9 & 0.8 & 12.8 & 1.0 & 13.4 & 1.2 & 14.1 & 0.9 \\
\hline 10.7 & 0.8 & 11.6 & 0.7 & 12.5 & 1.0 & 13.1 & 1.2 & 13.7 & 0.9 \\
\hline 9.9 & 0.8 & 10.7 & 0.8 & 11.5 & 0.9 & 12.3 & 1.1 & 12.9 & 0.9 \\
\hline 13.0 & 1.1 & 13.9 & 0.9 & 15.1 & 1.0 & 15.9 & 1.6 & 16.7 & 1.0 \\
\hline 10.7 & 1.4 & 11.3 & 1.3 & 12.0 & 1.6 & 12.8 & 1.5 & 12.8 & 1.7 \\
\hline 15.9 & 1.1 & 16.8 & 1.0 & 17.8 & 1.5 & 18.8 & 1.6 & 19.4 & 1.4 \\
\hline 16.6 & 1.3 & 17.7 & 1.1 & 18.9 & 1.4 & 19.9 & 1.5 & 20.6 & 1.3 \\
\hline 13.9 & 1.1 & 14.8 & 1.0 & 15.7 & 1.3 & 16.7 & 1.4 & 17.2 & 1.3 \\
\hline 19.4 & 1.3 & 20.5 & 1.2 & 22.1 & 1.6 & 23.1 & 1.8 & 24.0 & 1.5 \\
\hline 25.2 & 1.4 & 26.6 & 1.5 & 28.4 & 2.0 & 29.8 & 2.1 & 31.1 & 1.8 \\
\hline 27.1 & 1.5 & 28.6 & 1.5 & 30.7 & 2.0 & 32.0 & 2.1 & 33.7 & 1.9 \\
\hline 24.3 & 1.4 & 25.8 & 1.3 & 27.6 & 1.9 & 29.1 & 2.4 & 30.3 & 1.7 \\
\hline 17.6 & 1.0 & 18.7 & 1.3 & 20.0 & 1.7 & 21.4 & 1.9 & 22.4 & 1.5 \\
\hline 29.6 & 2.4 & 32.0 & 2.2 & 34.6 & 2.6 & 36.4 & 2.6 & 38.4 & 2.4 \\
\hline 32.9 & 2.6 & 35.2 & 2.3 & 37.8 & 2.7 & 39.6 & 2.6 & 42.1 & 2.4 \\
\hline 36.6 & 2.6 & 39.2 & 2.5 & 42.3 & 3.0 & 44.3 & 2.9 & 46.7 & 3.0 \\
\hline 38.4 & 2.6 & 41.1 & 2.5 & 44.5 & 2.8 & 46.7 & 2.9 & 49.1 & 2.7 \\
\hline 25.7 & 1.7 & 27.4 & 1.7 & 30.0 & 2.6 & 31.5 & 2.3 & 33.5 & 2.0 \\
\hline
\end{tabular}


Table 2B. Means and standard deviations (S.D.) for the lengths of the 19 tubu-

\begin{tabular}{|c|c|c|c|c|c|c|c|c|c|}
\hline \multirow{2}{*}{ Bones } & & \multicolumn{2}{|c|}{9} & \multicolumn{2}{|c|}{10} & \multicolumn{2}{|c|}{11} & \multicolumn{2}{|c|}{12} \\
\hline & & & S.D. & Mean & S.D. & Mean & S.D. & Mean & S.D. \\
\hline \multirow[t]{5}{*}{ Distal } & 5 & 12.8 & 1.1 & 13.7 & 1.0 & 14.2 & 1.4 & 15.0 & 1.3 \\
\hline & 4 & 14.9 & 1.0 & 15.6 & 1.1 & 16.5 & 1.4 & 17.1 & 1.4 \\
\hline & 3 & 14.5 & 1.0 & 15.4 & 1.3 & 16.2 & 1.4 & 16.6 & 1.3 \\
\hline & 2 & 13.4 & 1.0 & 14.3 & 1.1 & 15.0 & 1.2 & 15.5 & 1.1 \\
\hline & 1 & 17.8 & 1.2 & 18.6 & 1.4 & 19.7 & 1.6 & 20.3 & 1.7 \\
\hline \multirow[t]{4}{*}{ Middle } & 5 & 13.8 & 1.6 & 14.9 & 1.2 & 15.5 & 1.8 & 16.3 & 1.6 \\
\hline & 4 & 20.3 & 1.4 & 21.4 & 1.5 & 22.3 & 2.2 & 23.5 & 1.9 \\
\hline & 3 & 21.4 & 1.4 & 22.7 & 1.6 & 23.9 & 1.8 & 24.8 & 1.7 \\
\hline & 2 & 18.0 & 1.3 & 18.9 & 1.4 & 19.9 & 1.5 & 20.8 & 1.5 \\
\hline \multirow[t]{5}{*}{ Proximal } & 5 & 25.1 & 1.6 & 26.1 & 1.6 & 28.0 & 1.8 & 29.3 & 2.2 \\
\hline & 4 & 32.4 & 1.9 & 33.7 & 2.1 & 36.0 & 2.6 & 37.5 & 2.7 \\
\hline & 3 & 35.0 & 2.0 & 36.2 & 2.2 & 38.6 & 2.6 & 40.1 & 2.8 \\
\hline & 2 & 31.4 & 1.9 & 32.5 & 2.1 & 34.7 & 2.1 & 36.0 & 3.0 \\
\hline & 1 & 23.5 & 1.8 & 24.7 & 1.9 & 26.4 & 2.1 & 27.8 & 2.3 \\
\hline \multirow[t]{5}{*}{ Metacarpal } & 5 & 39.9 & 2.6 & 41.9 & 3.2 & 44.4 & 3.1 & 45.4 & 5.3 \\
\hline & 4 & 43.4 & 2.8 & 45.3 & 2.8 & 48.3 & 3.4 & 49.7 & 3.8 \\
\hline & 3 & 48.8 & 3.3 & 50.5 & 3.0 & 54.2 & 3.8 & 55.9 & 4.6 \\
\hline & 2 & 51.5 & 3.3 & 53.2 & 3.2 & 56.6 & 4.6 & 59.1 & 4. 2 \\
\hline & 1 & 34.7 & 2.3 & 35.9 & 2.6 & 38.9 & 2.7 & 40.3 & 3.0 \\
\hline \multirow[t]{5}{*}{ Distal } & 5 & 13.2 & 1.1 & 14.1 & 1.0 & 14.5 & 1.1 & 15.1 & 1.0 \\
\hline & 4 & 15.0 & 1.1 & 15.8 & 1.2 & 16.3 & 1.1 & 17.1 & 1.0 \\
\hline & 3 & 14.7 & 1.1 & 15.5 & 1.2 & 16.0 & 1.1 & 16.8 & 1.0 \\
\hline & 2 & 13.7 & 1.0 & 14.6 & 1.1 & 14.9 & 1.0 & 15.5 & 1.0 \\
\hline & 1 & 17.9 & 1.4 & 19.0 & 1.3 & 19.6 & 1.3 & 20.6 & 1.5 \\
\hline \multirow[t]{4}{*}{ Middle } & 5 & 13.9 & 2.1 & 15.2 & 1.7 & 15.9 & 1.5 & 16.8 & 2.2 \\
\hline & 4 & 20.6 & 1.8 & 22.1 & 1.7 & 23.1 & 1.3 & 24.5 & 1.5 \\
\hline & 3 & 21.9 & 1.9 & 23.5 & 1.8 & 24.4 & 1.4 & 26.0 & 1.8 \\
\hline & 2 & 18.3 & 1.7 & 19.8 & 1.6 & 20.5 & 1.3 & 21.9 & 1.3 \\
\hline \multirow[t]{5}{*}{ Proximal } & 5 & 25.6 & 2.0 & 27.5 & 1.9 & 28.9 & 1.6 & 30.7 & 1.7 \\
\hline & 4 & 32.9 & 2.2 & 35.2 & 2.4 & 36.9 & 2.0 & 39.0 & 2.1 \\
\hline & 3 & 35.4 & 2.4 & 38.0 & 2.5 & 39.7 & 2.1 & 42.2 & 2.6 \\
\hline & 2 & 31.9 & 2.1 & 34.3 & 2.3 & 35.9 & 1.9 & 38.0 & 2.1 \\
\hline & 1 & 24.0 & 2.2 & 26.1 & 2.2 & 27.3 & 1.7 & 29.0 & 1.7 \\
\hline \multirow[t]{5}{*}{ Metacarpal } & 5 & 40.5 & 3.3 & 43.3 & 2.7 & 45.2 & 2.7 & 47.6 & 2.6 \\
\hline & 4 & 44.1 & 3.1 & 47.1 & 2.9 & 48.9 & 2.6 & 51.6 & 2.7 \\
\hline & 3 & 49.5 & 3.6 & 52.7 & 3.3 & 54.9 & 3.0 & 58.3 & 3.5 \\
\hline & 2 & 52.1 & 3.8 & 55.6 & 3.5 & 57.4 & 4.2 & 61.5 & 3.4 \\
\hline & 1 & 35.4 & 2.7 & 37.8 & 2.5 & 39.5 & 2.1 & 41.5 & 2.0 \\
\hline
\end{tabular}

Jpn. J. Human Genet. 
Iar bones related to age (9-17 years) and sex (upper half, boys; lower half, girls).

\begin{tabular}{|c|c|c|c|c|c|c|c|c|c|}
\hline & \multicolumn{2}{|c|}{14} & \multicolumn{2}{|c|}{15} & \multicolumn{2}{|c|}{16} & \multicolumn{2}{|l|}{17} \\
\hline Mean & S.D. & Mean & S.D. & Mean & S.D. & Mean & S.D. & Mean & S.D. \\
\hline \multicolumn{10}{|l|}{ Boys } \\
\hline 16,0 & 1.4 & 17.0 & 1.2 & 17.2 & 1.2 & 17.7 & 1.1 & 17.6 & 1.5 \\
\hline 18.3 & 1.5 & 19.0 & 1.1 & 19.4 & 1.2 & 19.8 & 1.1 & 19.5 & 1.4 \\
\hline 17.9 & 1.4 & 18.5 & 1.1 & 18.9 & 1.1 & 19.1 & 1.2 & 19.0 & 1.5 \\
\hline 16.5 & 1.4 & 17.3 & 1.0 & 17.6 & 1.1 & 17.9 & 1.2 & 18.0 & 1.5 \\
\hline 22.0 & 2.0 & 22.9 & 1.5 & 23.2 & 1.4 & 23.3 & 1.6 & 23.5 & 1.9 \\
\hline 17.9 & 2.0 & 18.9 & 1.5 & 19.1 & 1.6 & 19.5 & 1.9 & 19.6 & 2.0 \\
\hline 25.6 & 2.1 & 26.5 & 1.5 & 26.6 & 1.6 & 27.2 & 1.7 & 27.5 & 1.9 \\
\hline 26.7 & 2.3 & 27.9 & 1.5 & 28.1 & 1.7 & 28.7 & 1.9 & 29.0 & 2. 0 \\
\hline 22.6 & 1.9 & 23.5 & 1.6 & 23.7 & 1.5 & 24.5 & 1.4 & 24.5 & 1.7 \\
\hline 31.7 & 2.6 & 33.1 & 2.1 & 34.0 & 2.0 & 34.8 & 1.9 & 34.6 & 2.4 \\
\hline 40.6 & 3.0 & 42.1 & 2.5 & 42.6 & 2.5 & 43.9 & 2.4 & 43.8 & 2.9 \\
\hline 43.1 & 3.2 & 45.3 & 2.5 & 45.6 & 2.7 & 46.8 & 2.6 & 46.6 & 3.2 \\
\hline 39.0 & 2.8 & 40.7 & 2.3 & 40.9 & 2.3 & 42.2 & 2.3 & 41.7 & 2. 7 \\
\hline 30.4 & 2.6 & 31.5 & 2.0 & 32.0 & 1.9 & 32.7 & 1.9 & 33.1 & 2.7 \\
\hline 49.6 & 3.6 & 51.6 & 2.8 & 52.9 & 3.0 & 54.2 & 3.1 & 53.5 & 3.6 \\
\hline 53.6 & 4.0 & 55.7 & 3.2 & 56.6 & 3.3 & 57.9 & 2.9 & 57.4 & 3.9 \\
\hline 60.4 & 4.5 & 63.1 & 4.0 & 63.9 & 3.9 & 65.4 & 3.6 & 64.9 & 4.4 \\
\hline 63.5 & 4.6 & 66.2 & 3.7 & 67.0 & 3.9 & 68.4 & 4.2 & 67.5 & 4.4 \\
\hline \multicolumn{10}{|l|}{ Girls } \\
\hline 15.6 & 1.2 & 15.6 & 1.0 & 15.9 & 1.3 & 16.0 & 1.2 & 15.8 & 1.2 \\
\hline 17.7 & 1.0 & 17.6 & 0.9 & 17.7 & 1.1 & 18.0 & 1.1 & 17.8 & 1.3 \\
\hline 17.2 & 1.0 & 17.1 & 1.0 & 17.3 & 1.1 & 17.5 & 1.0 & 17.4 & 1.1 \\
\hline 16.1 & 1.0 & 15.9 & 0.9 & 16.2 & 1.1 & 16.5 & 0.9 & 16.3 & 1.1 \\
\hline 21.1 & 1.4 & 20.9 & 1.1 & 21.1 & 1.5 & 21.3 & 1.2 & 21.3 & 1.5 \\
\hline 17.1 & 1.8 & 17.3 & 1.6 & 17.3 & 1.8 & 17.5 & 1.8 & 17.5 & 1.6 \\
\hline 24.6 & 1.8 & 25.0 & 1.3 & 25.2 & 1.5 & 25.2 & 1.3 & 25.0 & 1.7 \\
\hline 26.1 & 2.0 & 26.4 & 1.3 & 26.4 & 1.7 & 26.8 & 1.6 & 26.5 & 1.3 \\
\hline 21.9 & 1.7 & 22.2 & 1.2 & 22.2 & 1.3 & 22.4 & 1.1 & 22.4 & 1.4 \\
\hline 31.5 & 1.6 & 31.6 & 1.4 & 32.0 & 1.6 & 32.0 & 1.6 & 32.0 & 2.0 \\
\hline 40.0 & 2.1 & 40.2 & 1.7 & 40.5 & 2.1 & 40.4 & 1.8 & 40.4 & 2.3 \\
\hline 42.4 & 2.4 & 42.9 & 1.7 & 43.3 & 2.3 & 43.3 & 1.9 & 43.0 & 2.1 \\
\hline 38.6 & 2.3 & 38.8 & 1.6 & 38.9 & 2.0 & 39.0 & 1.8 & 38.9 & 2. 2 \\
\hline 29.5 & 1.7 & 29.7 & 1.5 & 29.9 & 1.8 & 29.9 & 1.5 & 30.1 & 1.9 \\
\hline 48.7 & 2.4 & 49.3 & 2.1 & 50.0 & 2.8 & 50.0 & 2.6 & 49.3 & 3.2 \\
\hline 53.0 & 2.5 & 52.9 & 2.0 & 54.0 & 3.3 & 53.7 & 2.7 & 53.2 & 3.2 \\
\hline 60.0 & 2.9 & 60.1 & 2.7 & 60.8 & 3.7 & 60.4 & 3.3 & 60.3 & 3.7 \\
\hline 62.5 & 2.5 & 63.2 & 2.7 & 63.9 & 3.5 & 63.2 & 3.3 & 63.0 & 3.7 \\
\hline 41.9 & 2.2 & 42.5 & 2.0 & 42.6 & 2.3 & 43.0 & 2.1 & 42.4 & 2.7 \\
\hline
\end{tabular}




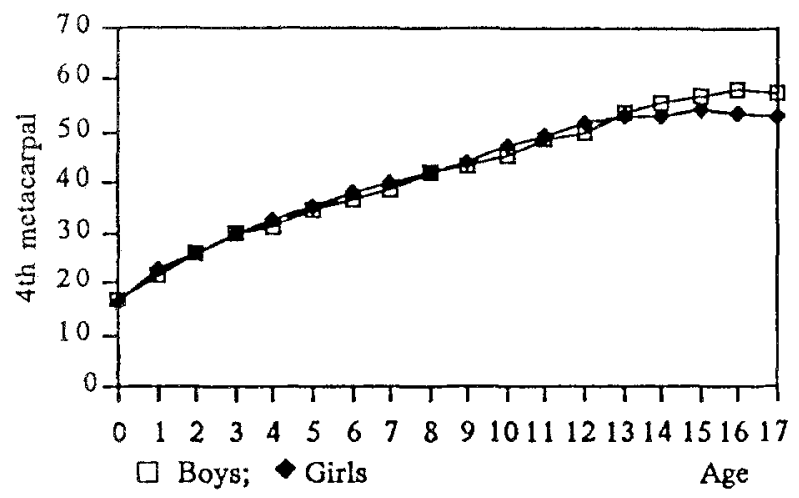

Fig. 2. The growth of the 4th metacarpal bone of the Japanese.
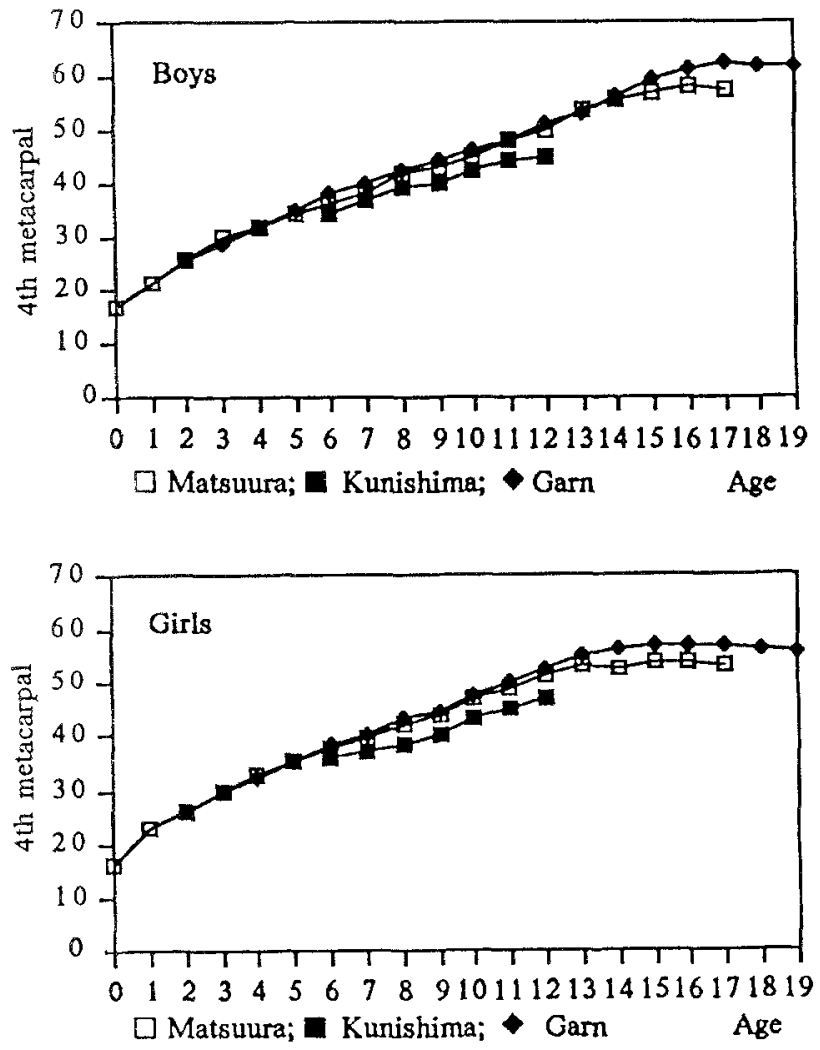

Fig. 3. Comparison between Japanese and Caucasian children. $\square$, Matsuura, the present study; $\square$, Kunishima, 711 Japanese children of 6-12 years of age reported by Kunishima (1965); $\$$, Garn, Caucasian children of 2-19 years of age reported by Garn et al. (1972). 
bones of the hand of children grouped according to sex and yearly intervals. The $t$-test revealed no statistically significant regional difference at the $5 \%$ level in the lengths of the 19 tubular bones in 4- and 6-year-old children from Nagoya, Saga and Nagasaki.

Sex-associated differences were found in the growth of the 19 tubular bones. There was a tendency for boys to exceed girls at the 14 to 17 year intervals. This is illustrated in Fig. 2, showing the 4th metacarpal as an example.

Figures $3 a$ and $3 b$ show sex- and age-specific means of the 4th metacarpal lengths in our data (Matsuura) sampled between 1976 and 1985 in three separate areas of Japan, those of Japanese children collected in 1959 and 1960 in Shizuoka city (Kunishima, 1965), and those of Caucasian children (Garn et al., 1972). Our data in the 6 to 12 year range show mean lengths longer than those by Kunishima (1965). This would reflect accelerated growth in Japanese children that occurred during the interval between the two studies, rather than regional differences. Overall, our data are close to those of Caucasian children until the age of 15 years in boys and 13 years in girls. Thereafter, Japanese figures level off, while Caucasian children keep growing a few more years. The mean lengths of the 2 nd to 4 th distal phalanges in the 3 to 13 year range in our data are longer than those of Caucasian children.

\section{DISCUSSION}

The present study, dealing with 1,585 children of $0-17$ years of age studied during the period from 1976 to 1985 , would serve as the only standard available

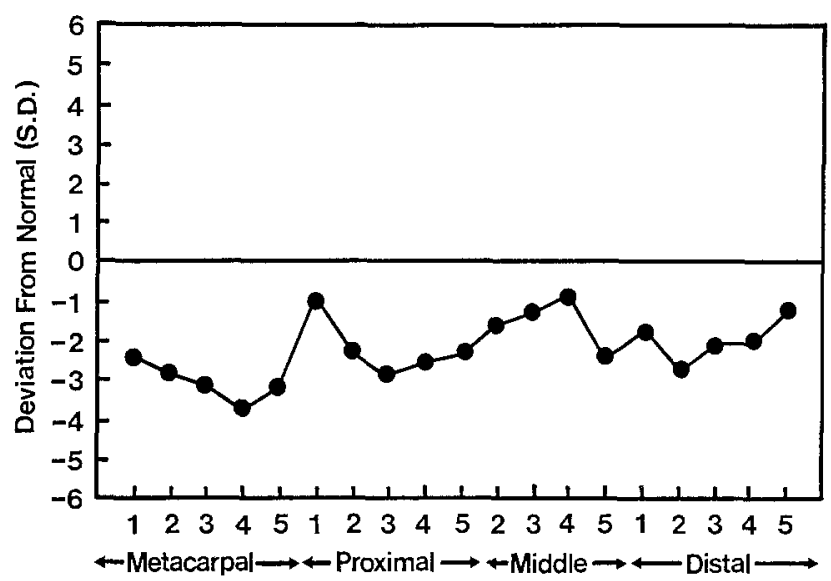

Fig. 4. Metacarpophalangeal pattern profile (MCPP) analysis of the 45, X patient shown in Fig. 1. Standard deviations of the bone lengths from the mean values are displayed, with standard deviations on the ordinate and the 19 tubular bones, sequentially arrayed, on the abscissa. Overall shortening of the hand tubular bones, especially of the 4th metacarpal, and a relatively long proximal phalanx of the thumb. 
for today's Japanese children. In the present study, the number of children in each group in the 0 to 3 year range was 9 to 12 while it was 49 to 66 in the 4 to 17 year range.

An example of metacarpophalangeal pattern profile analysis is being presented using the hand radiograph in Fig. 1 as the subject (Fig. 4). The patient, a 16-yearold girl with a 45,X karyotype, revealed overall shortened hand tubular bones, and relative lengthening of the proximal phalanx of the thumb.

Acknowiedgments We wish to thank Dr. Y. Sugiura, Nishio Municipal Hospital, and Prof. T. Akiyoshi, Department of Anatomy, Saga Medical Coliege, for allowing us to analyze the radiographs in their respective collection. Our thanks are also due to the advice of Dr. M. Tsukahara, and the help of Misses K. Shibata and K. Nakajima.

\section{REFERENCES}

Al-Awadi, S.A., Farag, T.I., Satyanath, S. and Sundareshan, T.S. 1988. Letter to the editor: Metacarpophalangeal pattern profile analysis in partial 9p monosomy. Am. J. Med. Genet. 29: 217-219.

Butler, M.G., Meaney, F.J., Kittur, S., Hersh, J.H. and Hornstein, L. 1985a. Metacarpophalangeal pattern profile analysis in Sotos syndrome. Am. J. Med. Genet. 20: 625-629.

Butler, M.G. and Meaney, F.J. 1985b. Metacarpophalangeal pattern profile analysis in PraderWilli Syndrome, a follow-up report on 38 cases. Clin. Genet. 28: 27-30.

Butler, M.G., Meaney, F.J. and Kaler, S.G. 1986. Metacarpophalangeal pattern profile analysis in clinical genetics: An applied anthropometric method. Am. J. Phys. Anthropol. 70: 195201.

Butler, M.G., Gale, D.D., Meaney, F.J., Wadlington, W.B. and Robinow, M. 1987a. Metacarpophalangeal pattern profile analysis in Robinow syndrome. Am. J. Med. Genet. 27: 219-223.

Butler, M.G., Gale, D.D. and Meaney, F.J. 1987b. Metacarpophalangeal pattern profile analysis in diastrophic dysplasia. Am. J. Med. Genet. 28: 685-689.

Butler, M.G., Rames, L.J. and Wadlington, W.B. 1988. Acrodysostosis: Report of a 13-year-old boy with review of literature and metacarpophalangeal pattern profile analysis. Am. J. Med. Genet. 30: 971-980.

Garn, S.M., Hertzog, K.P., Poznanski, A.K. and Nagy, J.M. 1972. Metacarpophalangeal length in the evaluation of skeletal malformation. Radiology 105: 375-381.

Hasue, M., Kato, T., and Watanabe, T. 1955. On the lengths of the metacarpals and phalanges J. Nihon Univ. Med. Assoc. (in Japanese) 14: 1538-1545.

Igarashi, M. and Kajii, T. 1988. Normal values for physical parameters of the head, face and hand in Japanese children. $J_{p n}$. J. Human Genet. 33: 9-31.

Kato, T., Hasue, M. and Sato, M. 1955. On the growth patterns of the metacarpals and phalanges in children. J. Nihon Univ. Med. Assoc. (in Japanese) 14: 1531-1537.

Kunishima, Y. 1965. Roentgenologic study on the lengths of phalanges and metacarpals among bealthy school children. Chubu Nihon Seikeigeka Saigaigeka Gakkai Zasshi (in Japanese) 8: $377-384$.

Poznanski, A.K., Garn, S.M., Nagy, J.M. and Gall, J.C. 1972. Metacarpophalangeal pattern profiles in the evaluation of skeletal malformations. Radiology 104: 1-11.

Poznanski, A.K. 1983. Pattern profile comparisons: Differences and similarities. Ann. Radiol. 27: 89-96.

Sugiura, Y. 1985. Skeletal Age of the Japanese (in Japanese), Chugai Igaku, Tokyo.

Takai, S. and Akiyoshi, T. 1983. Skeletal maturity of Japanese children in Western Kyushu. Am. J. Phys. Anthropol. 62: 199-204.

Tonoki, H., Ohura, T. and Niikawa, N. 1988. Cryptomicrotia and short, stubby fingers with excess fingertip arch patterns in a mother and son. Am. J. Med. Genet. 29: 857-862. 